\title{
Synthesis of novel water soluble onium salts and thieno[3,4- c]thiolactones - precursors of conductive materials derived from substituted 2-aminothiophenes
}

\author{
Zita Puterováa $^{a}$, Daniel Végh, ${ }^{a^{*}}$ Renata Gottasová, ${ }^{a}$ and Zsolt Végh ${ }^{b}$ \\ a Department of Organic Chemistry, Faculty of Chemical Technology, Slovak University of \\ Technology, Radlinského 9, SK - 81237 Bratislava, Slovak Republic \\ b VUCHT joint stock company, Bratislava SK \\ E-mail: daniel.vegh@stuba.sk
}

Dedicated to Professor Rosa Lederkremer on her $70^{\text {th }}$ anniversary

\begin{abstract}
The synthetic utility in the area of synthesis of "smart" conductive materials of the easily accessible 2-aminothiophenes is described. Simple protection of amino group and subsequent radical bromination leads to brominated intermediates. The novel 4-bromomethylthiophene derivatives easily enter into reaction with tertiary amines and thiourea to give ammonium and thiouronium salts - novel water-soluble thiophenes. Thiouronium salts are predisposed to deliver substituted thieno[3,4-c] thiolactone derivatives, which are attractive from the point of view of their further utilization in the synthesis of thieno[3,4-c]thiophenes. Each of the mentioned compounds showed bioactivity as well as propensity to be polymerized to conductive oligomers and polymers.
\end{abstract}

Keywords: 2-Aminothiophenes, thieno[3,4-c]thiolactones, thieno[3,4-c] thiophenes ammonium salts, water soluble conductive materials

\section{Introduction}

Functionalized polyheterocycles belong to a class of novel polyconjugated materials possessing unconventional electrical, optical and magnetic properties. ${ }^{1}$ Substituted polythiophenes are important members of this family, especially the poly-3- and 3,4-substituted ones. ${ }^{2,3}$ Thiophene derivatives are the most promising materials for a variety of applications in electronics and optoelectronics. They are unique for their relative stability, suitable optical nonlinearity and spectral characteristics. ${ }^{4}$ In addition to being environmentally stable; they possess good workability and satisfactory solubility in both organic and aqueous media. Their broad range of 
properties makes them suitable for a new generation of useful tools in the areas of diagnostics, therapeutics, drug screening.

The aim of our study was to synthesize new ammonium salts (Scheme 1) from the corresponding bromothiophene derivatives by their reaction with tertiary amines in a simple manner. Ammonium salts are water soluble compounds with predictable biological activity.

We were also interested in the synthesis of thieno[3,4-c]thiolactone derivates (Scheme 2). Thieno[3,4-c] thiolactones seem to be useful intermediates in the synthesis of thieno[3,4$c]$ thiophenes. Such derivatives represents a $10-\pi$ electron heteropentalene system containing nonclassical tetracovalent sulphur nucleus and are have been investigated from the synthetic and theoretical point of view; ${ }^{5-7}$ they also represents important building blocks in the synthesis of polymeric conductive materials.

\section{Results and Discussion}

The substituted 2-aminothiophenes (1a-c) represent an easily available starting material for the synthesis of target thieno[3,4-c]thiolactone derivatives (7a-c). Preparation of 2-aminothiophenes is a very simple, one-step reaction originally published by Gewald. ${ }^{8}$ We have now synthesized three types of 2-aminothiophenes by two of procedures. In the first one we prepared 5unsubstituted 2-aminothiophene derivative 1a by the procedure published in our earlier paper. ${ }^{9}$ Persubstituted 2-aminothiophenes 1b,c were prepared afforded the appropriate 2aminothiophenes in good yields and purity. Simple protection of amino group, necessary for next step bromination, and performed by acetic anhydride with catalytic use of $\left(\mathrm{MgClO}_{4}\right)_{2} .2 \mathrm{H}_{2} \mathrm{O}$ led to $N$-acetylaminothiophene derivatives (2a-c).

The methyl group of $\mathrm{N}$-acetylaminothiophenes 2a-c could be successfully brominated. For bromination of methyl 2-acetylamino-4-methyl-3-thiophene carboxylate (2a) two approaches were tried, one by bromine in acetic acid, the other by $N$-bromosuccinimide in carbon tetrachloride (Scheme 1). In the first method two equivalents of bromine were used, and bromination took place at the methyl group in position $\mathrm{C}-4$ as well to at position C-5 to give the 5-bromo-4-bromomethyl derivative 3a in $90 \%$ yield. In the second approach, the use of $N$ bromosuccinimide in carbon tetrachloride, a typical radical bromination of side chains with use of dibenzoyl peroxide as an initiator, failed to produce bromomethylated derivative without bromine at the $\mathrm{C}-5$ position of thiophene. In this case the thiophene position 5 was the first to be brominated, giving a monobrominated product, followed by bromination of the methyl group and the target bromomethylene thiophene derivate 3a, isolated from the reaction mixture with monobrominated derivate. Only the use of 2 equiv. of $N$-bromosuccinimide, 4-5 refluxing in carbon tetrachloride pushed the yield of 3a to the maximum of $69 \%$. However, the use of $N$ bromosuccinimide in $\mathrm{CCl}_{4}$ (with dibenzoylperoxide) means a simple and successful method for brominating the methyl group in a case of 5-acetylamino-3-methylthiophene-2,4-dicarboxylic acid dimethylester (2b) and its 2-tert-butoxycarbonyl analogue (2c) (Scheme 2) to give 
bromoderivates 3b,c in approximately yield $70 \%$. Bromomethylated derivatives of 2 aminothiophnenes (3a-c) are key intermediates in the synthesis of quaternary ammonium salts 4a-d (Scheme 1) and the thiouronium salts 5a-c (Scheme 2).

Quaternary ammonium salts 5a-c were prepared by the treatment of methyl 2-acetylamino-4bromomethyl-5-bromothiophene-3-carboxylate (3a) with tertiary amines in toluene (Scheme 1) at laboratory temperature. The target compounds were prepared in yields $40-90 \%$ and were characterized by UV, IR and ${ }^{1} \mathrm{H}$ NMR spectra (for all compounds listed in experimental section). The UV spectra of each quaternary ammonium salts showed characteristic absorption maximum at 220 and $300 \mathrm{~nm}$. The characteristic signal of methylene group in ${ }^{1} \mathrm{H}$ NMR spectra was at 4-6 ppm. Quaternary ammonium salts $\mathbf{5 a - c}$ are predisposed to be biological active compounds.

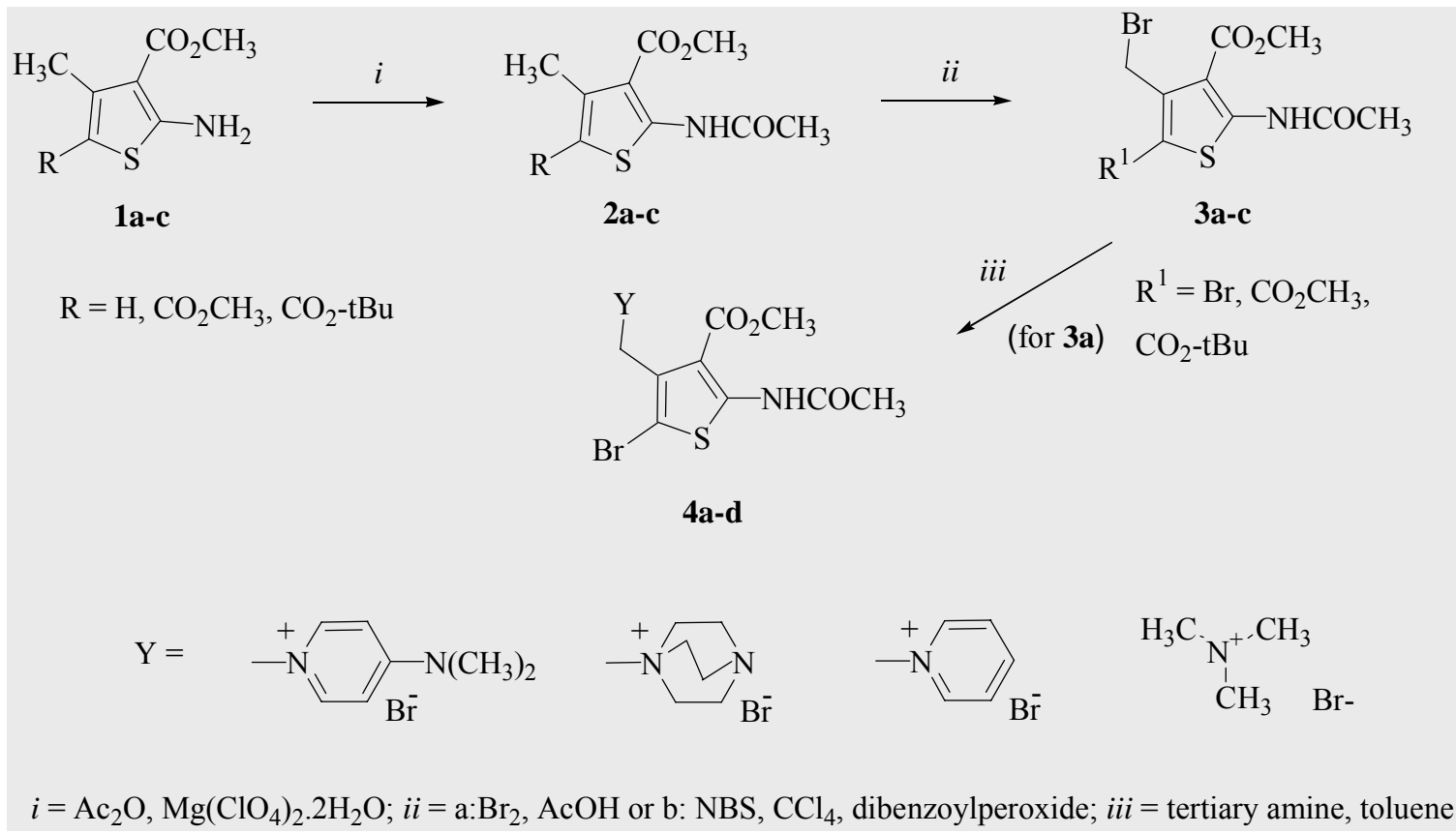

\section{Scheme 1}

The thiouronium salts 5a-c were prepared in a high yields (91-95\%) by the reaction of the corresponding bromomethylated aminothiophenes 3a-c with thiourea in dry acetone under inert argon atmosphere (Scheme 2). Final salts are stable compounds soluble in aqueous media, which in the next step were decomposed by aqueous $\mathrm{NaHCO}_{3}$ to give the target thieno[3,4-c] thiolactone derivatives 7a-c. The hydrolytic decomposition was performed in two manners (Scheme 2). When thiouronium salt 5 a was refluxed in saturated aqueous solution of $\mathrm{NaHCO}_{3}$, a mixture of three compounds was isolated from the reaction mixture - the desired thieno[3,4-c]tiolactone derivative 7a with 5-bromo-4-sulfanylmethylthiophene-3-carboxylic acid $\mathbf{6 a}$ and its methylester 8a. To simplify the characterisation of the mixture of mentioned three compounds the sulfanylmetylderivate $\mathbf{6 a}$ was also prepared by hydrolysis in methanolic $\mathrm{KOH}^{11}$ to help identify it 
in the mixture of 6a, 7a and 8a. Products were separated by flash column chromatography and characterised by IR and ${ }^{1} \mathrm{H}$ NMR spectra.

In the second approach to hydrolytic decomposition of thiouronium salts to get thieno[3,4c]thiolactone derivatives we used the method developed by Pal and coworkers. For the cyclisation of thiouronium salt 5a-c we modified $\mathrm{Pal}^{\prime} \mathrm{s}^{12}$ reaction conditions and $1 \mathrm{M}$ methanolic solution of $\mathrm{NaHCO}_{3}\left(50 \%\right.$ methanol in water) was used and the mixture heated at $70-80^{\circ} \mathrm{C}$ under inert atmosphere of argon. However, the yields were low (only 40-50\%), only the desired thieno[3,4-c] thiolactone derivates 7a-c were isolated after reaction. In our opinion unreacted thiouronium salts and supplementary products remain completely dissolved in water, while the corresponding thiolactones, being water insoluble compounds, can be easily separated and isolated from the crude mixture.

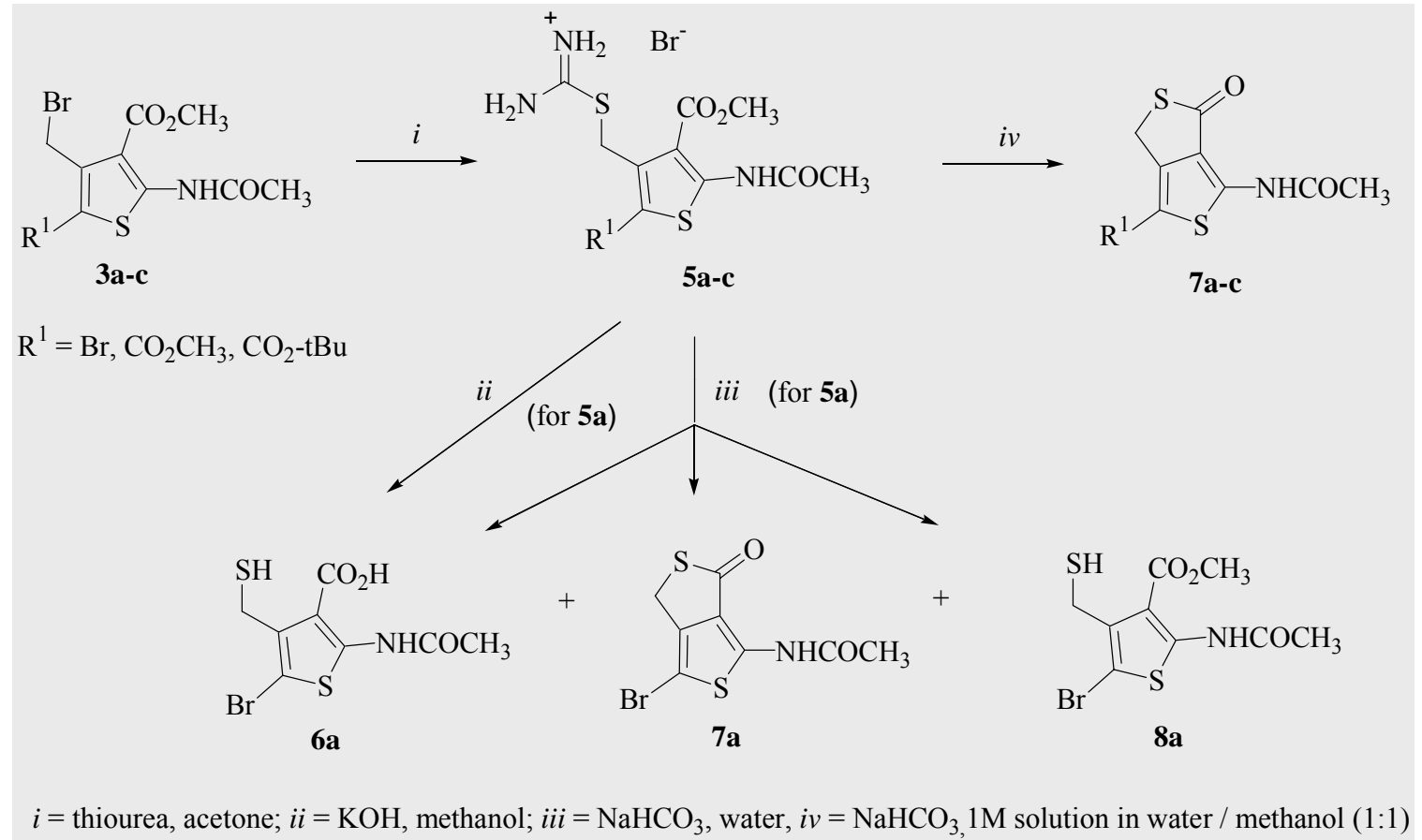

\section{Scheme 2}

The IR, ${ }^{1} \mathrm{H}$ NMR and ${ }^{13} \mathrm{C}$ NMR spectral data are collated in the Experimental section. The ${ }^{1} \mathrm{H}$ NMR spectra display singlets for methylene group in the $4.50-4.90 \mathrm{ppm}$ region for thiouronium salts 5a-c and in the area 4.30-.50 ppm region for thieno[3,4-c]thiophenes 7a-c. In the ${ }^{13} \mathrm{C}$ NMR spectra the chemical shift for carbonyl of methylene group is in the area $30-5 \mathrm{ppm}$. The signal for $\mathrm{C}=\mathrm{O}$ group of new designed thiolactones $7 \mathbf{a}-\mathbf{c}$ is in the area $180-90 \mathrm{ppm}$. It is a significant shift for cyclic lactone derivatives. ${ }^{13} \mathrm{C}$ chemical shifts, given in the experimental section, assignments were straightforward using gs-HSQC (gradient selected Heteonuclear Quantum Coherence) and gs- HMBC (gradient selected Heteronuclear Multiple Bond correlation) spectroscopy. 


\section{Experimental Section}

General Procedures. All solvents were distilled before use. Melting points were determined with the Kofler hot stage and are uncorrected. Flash column liquid chromatography was performed on silica gel Kiesegel 60 (40-63 $\mu \mathrm{m}, 230-400$ mesh) and analytical thin-layer chromatography (TLC) was performed on aluminium pre-coated with $0.2 \mathrm{~mm}$ silica gel (DCAlufolien, Merck). The compounds were visualised by UV lamp at $254 \mathrm{~nm}$ wavelength or by dipping the plates in iodine solution. ${ }^{1} \mathrm{H}-\mathrm{NMR}(300 \mathrm{MHz})$ and ${ }^{13} \mathrm{C}-\mathrm{NMR}(75 \mathrm{MHz})$ spectra were recorded on a Varian VXR 300 instrument at $293^{\circ} \mathrm{K}$ in $\mathrm{CDCl}_{3}$. DMSO-d $d_{6}$. Spectra were internally referenced to TMS. Chemical shifts $(\delta$-scale) are quoted in parts per million (ppm) and the following abbreviations are used: singlet (s), doublet (d), triplet (t), quartet (q), broad singlet (brs) some combinations of these were made by DEPT editing of the spectra. Infrared spectra in the region 400-4000 $\mathrm{cm}^{-1}$ were taken with Philips Analytical PU9800 FTIR spectrophotometer as $\mathrm{KBr}$ disks $(0,3 \mathrm{mg}$ in $300 \mathrm{mg}$ of $\mathrm{KBr})$. Elemental analyses ${ }^{13}$ were measured on an Carlo Erba, Milan type instrument. Compounds are numbered according to Schemes 1,2.

Materials. All solvents were distilled before use. Commercial reagents were used without further purification. 2-Amino-4-methylthiophene-3-carboxylic acid methyl ester (1a) and 2Acetylamino-3-methylthiophene-carboxylic acid methyl ester (2a) were prepared in the same manner as is published in our previous paper. ${ }^{9}$

5-Amino-3-methylthiophene-2,4-dicarboxylic acid dimethyl ester (1b) according to the procedure described by Gudriniece, ${ }^{10}$ a mixture of sulphur $(14 \mathrm{~g})$ and morpholine $(60 \mathrm{~mL})$ was vigorously stirred in a three necked flask at $110^{\circ} \mathrm{C}$ for 3 hours. After cooling down to laboratory temperature, the mixture of methyl acetoacetate $(4.64 \mathrm{~g}, 40 \mathrm{mmol})$ and methylcyanoacetate $(3.96$ $\mathrm{g}, 40 \mathrm{mmol})$ in methanol $(100 \mathrm{~mL})$ was added dropwise to morpholinepolysulfide prepared in situ. The reaction solution was mixed for further 24 hours at laboratory temperature. Resulting yellow powdered product $(5.5 \mathrm{~g}, 60 \%)$ was filtered of in satisfactory purity for use in consecutive reaction. M.p. $=147-149^{\circ} \mathrm{C} .{ }^{1} \mathrm{H}-\mathrm{NMR}\left(\mathrm{DMSO}-\mathrm{d}_{6}, 300 \mathrm{MHz}\right) \delta 2.66\left(\mathrm{~s}, 3 \mathrm{H}, \mathrm{CH}_{3}\right), 3.79(\mathrm{~s}, 3 \mathrm{H}$, $\mathrm{CO}_{2} \mathrm{CH}_{3}$ ), $3.84\left(\mathrm{~s}, 3 \mathrm{H}, \mathrm{CO}_{2} \mathrm{CH}_{3}\right), 6.58\left(\mathrm{brs}, 2 \mathrm{H}, \mathrm{NH}_{2}\right) ;{ }^{13} \mathrm{C}-\mathrm{NMR}$ (DMSO-d, $\left.75 \mathrm{MHz}\right) \delta 15.39$ $\left(\mathrm{CH}_{3}\right), 50.53,51.03\left(2 \mathrm{xCO}_{2} \mathrm{CH}_{3}\right), 108.09,110.02,147.51,150.2$ (C-2, C-3, C-4, C-5), 164.82, $166.54\left(2 \mathrm{xCO}_{2} \mathrm{CH}_{3}\right)$.

5-Amino-3-methylthiophene-2,4-dicarboxylic acid 2-tertbutylester-4-methylester (1c) was prepared according to the same procedure as $\mathbf{1 b}$.Yield $84 \%$ (9.1g white powdered product) M.p. $=134-137^{\circ} \mathrm{C} .{ }^{1} \mathrm{H}-\mathrm{NMR}\left(\mathrm{DMSO}-d_{6}, 300 \mathrm{MHz}\right) \delta 1.39\left(\mathrm{~s}, 9 \mathrm{H}, \mathrm{CO}_{2} \mathrm{C}\left(\mathrm{CH}_{3}\right)_{3}\right), 2.56\left(\mathrm{~s}, 3 \mathrm{H}, \mathrm{CH}_{3}\right)$, $3.73\left(\mathrm{~s}, 3 \mathrm{H}, \mathrm{CO}_{2} \mathrm{CH}_{3}\right.$ ), 7.79 (brs, $\left.2 \mathrm{H}, \mathrm{NH}_{2}\right) ;{ }^{13} \mathrm{C}-\mathrm{NMR}$ (DMSO-d 6 , 75MHz) $\delta 15.25\left(\mathrm{CH}_{3}\right), 27.83$

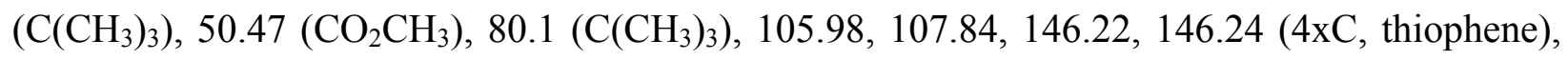
$164.85\left(\mathrm{CO}_{2} \mathrm{CH}_{3}\right), 166.18\left(\mathrm{CO}_{2}\left(\mathrm{CH}_{3}\right)_{3}\right)$.

5-Acetylamino-3-methylthiophene-2,4-dicarboxylic acid dimethyl ester (2b). 2Aminothiophenecarboxylate (1b) $(9.6 \mathrm{~g}, 42 \mathrm{mmol})$ in acetic anhydride and 2-3 crystals of $\mathrm{Mg}\left(\mathrm{ClO}_{4}\right)_{2} \cdot 2 \mathrm{H}_{2} \mathrm{O}$ were heated on silicon bath for 4 hours. The reaction mixture was then cooled 
down and left at laboratory temperature for 12 hours. The white needles of product were filtered on Büchner to get $75 \%$ (8.55g white needles) used without cleaning in the next step. M.p. $=177$ $181^{\circ} \mathrm{C} .{ }^{1} \mathrm{H}-\mathrm{NMR}\left(\mathrm{DMSO}-\mathrm{d}_{6}, 300 \mathrm{MHz}\right) \delta 2.61\left(\mathrm{~s}, 3 \mathrm{H}, \mathrm{CH}_{3}\right), 3.26\left(\mathrm{~s}, 3 \mathrm{H}, \mathrm{COCH}_{3}\right), 3.78(\mathrm{~s}, 3 \mathrm{H}$, $\mathrm{CO}_{2} \mathrm{CH}_{3}$ ), $3.86\left(\mathrm{~s}, 3 \mathrm{H}, \mathrm{CO}_{2} \mathrm{CH}_{3}\right), 11.07$ (brs, $\left.1 \mathrm{H}, \mathrm{NH}\right) ;{ }^{13} \mathrm{C} \mathrm{NMR}\left(\mathrm{DMSO}-d_{6}, 75 \mathrm{MHz}\right) \delta 14.61$ $\left(\mathrm{CH}_{3}\right), 23.0\left(\mathrm{COCH}_{3}\right), 51.46,51.74\left(2 \mathrm{xCO}_{2} \mathrm{CH}_{3}\right), 110.1,111.2,144.3,145.2$ (4xC, thiophene), $165.1,166.2\left(2 \mathrm{xCO}_{2} \mathrm{CH}_{3}\right)$.

5-Acetylamino-3-methylthiophene-2,4-dicarboxylic acid 2-tertbutylester-4-methylester (2c) was prepared in the same manner as $\mathbf{2 b}$. Yield 78\% (10.27g light brown crystals). M.p. = 204$207^{\circ} \mathrm{C} .{ }^{1} \mathrm{H}-\mathrm{NMR}\left(\mathrm{DMSO}-d_{6}, 300 \mathrm{MHz}\right) \delta 1.52\left(\mathrm{~s}, 9 \mathrm{H}, \mathrm{CO}_{2}\left(\mathrm{CH}_{3}\right)_{3}\right), 2.27\left(\mathrm{~s}, 3 \mathrm{H}, \mathrm{CH}_{3}\right), 3.21(\mathrm{~s}$, $\mathrm{COCH}_{3}$ ), 3.87 (s, 3H, $\mathrm{CO}_{2} \mathrm{CH}_{3}$ ), 11.03 (brs, $\left.1 \mathrm{H}, \mathrm{NH}\right) ;{ }^{13} \mathrm{C} \mathrm{NMR}$ (DMSO-d, $\left.75 \mathrm{MHz}\right) \delta 14.45$

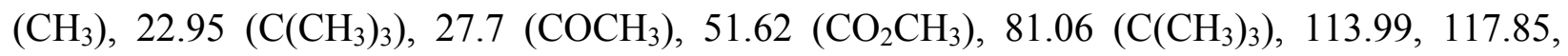

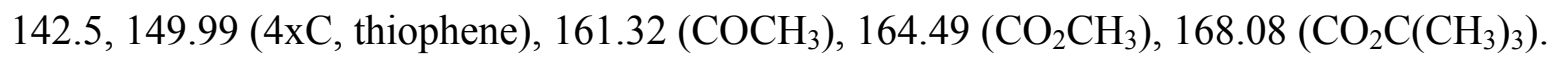

\section{2-Acetylamino-4-bromomethylthiophene-3-carboxylic acid methyl ester (3a)}

Method A. Bromine $(2.55 \mathrm{~mL}, 50 \mathrm{mmol})$ was added dropwise to methyl ester 2a (10.6 g, $49 \mathrm{mmol}$ ) dissolved in $100 \mathrm{~mL}$ of acetic acid in an apparatus kept under a slow argon flow. The mixture was gradually warmed up to reflux, the $\mathrm{HBr}$ was carried away from the top of the condenser to an absorber. After removing the $\mathrm{HBr}$ from reaction mixture (visually identified by loss of dark colour caused by unreacted bromine, after 5 hours), another 8 g portion of bromine (2.55 $\mathrm{mL}, 50 \mathrm{mmol}$ ) was added dropwise and the reaction mixture was heated to reflux for another 5 hours until $\mathrm{HBr}$ evolution had finished. The reaction mixture was washed with water solution of $\mathrm{Na}_{2} \mathrm{~S}_{2} \mathrm{O}_{7}$, then with water. Acetic acid was then evaporated to get and crude dark product. Crystallisation from ethyl acetate lead to $90 \%$ (17 g dark grey crystals) of 3a.

Method B. To the vigorously stirred mixture of $(8 \mathrm{~g}, 37.6 \mathrm{mmol})$ of methyl ester $2 \mathrm{a}, 1 \mathrm{~g}$ $(6 \mathrm{mmol})$ of dibenzoyl peroxide and $300 \mathrm{~mL}$ of $\mathrm{CCl}_{4}$ placed in a three-necked $500 \mathrm{~mL}$ flask was gradually added $N$-bromosuccinimide (NBS, 7.9g, 75.2mmol). The reaction mixture was heated to reflux on oil bath. The stirring continued for 4-5 hours at a boiling point of carbon tetrachloride. After cooling down to laboratory temperature the separated succinimide was removed by filtration under vacuum and the solvent was evaporated to dryness and the dark residue was completely dissolved in ethyl acetate. The solution was then refluxed for 30 min and cooled. The dark brown precipitate was isolated by filtration, washed with ethyl acetate to give $91 \%(10.9 \mathrm{~g})$ of crude mixture of $3 \mathbf{a}$ and

2-Acetylamino-4-methyl-5-bromothiophene-3-carboxylic acid methyl ester = 2.5: 1 The separation and purification was carried by flash column liquid chromatography, eluted by chloroform to obtain $62 \%$ (6.8g dark grey crystals) of 3a and $19 \%$ (2.7g light brown crystals) of monobrominated thiophene derivative (m.p. $\left.=106-109^{\circ} \mathrm{C}\right)$.

For 3a in each procedure M.p. $=156-160^{\circ} \mathrm{C} .{ }^{1} \mathrm{H}-\mathrm{NMR}\left(\mathrm{DMSO}-d_{6}, 300 \mathrm{MHz}\right) \delta 2.25(\mathrm{~s}, 3 \mathrm{H}$, $\mathrm{COCH}_{3}$ ), $3.86\left(\mathrm{~s}, 3 \mathrm{H}, \mathrm{CO}_{2} \mathrm{CH}_{3}\right), 4.75\left(\mathrm{~s}, 2 \mathrm{H}, \mathrm{CH}_{2}\right), 10.99$ (brs, $\left.1 \mathrm{H}, \mathrm{NH}\right) ;{ }^{13} \mathrm{C}-\mathrm{NMR}$ (DMSO- $d_{6}$, $75 \mathrm{MHz}) \delta 14.45\left(\mathrm{CH}_{2}\right), 27.7\left(\mathrm{COCH}_{3}\right), 51.62\left(\mathrm{CO}_{2} \mathrm{CH}_{3}\right), 113.99,117.85,142.5,149.99(4 \mathrm{xC}$, thiophene), $164.58\left(\mathrm{COCH}_{3}\right), 168.08\left(\mathrm{CO}_{2} \mathrm{CH}_{3}\right)$. 


\section{5-Acetylamino-3-bromomethyl-thiophene-2,4-dicarboxylic acid dimethyl ester (3b)}

To a solution of 3,5-Dimethyl-2-acetamino-4-methylthiophene-3,5-dicarboxylate (2b) (1.36g, $5 \mathrm{mmol})$ in carbon tetrachloride $12.5 \mathrm{~mL}$ containing dibenzoylperoxide as initiator $(1 \mathrm{mg}) \mathrm{N}$ bromosuccinimide $(0.9 \mathrm{~g}, 5 \mathrm{mmol})$ was added in one portion. The mixture was refluxed for 6 hours, until the starting materials completely disappeared (TLC controlled $40 \%$ of EtOAc in $i$ hexane). The mixture was cooled down to room temperature; succinimide was removed by filtration in vacuum. The filtrate was concentrated under reduced pressure and the crude product was crystallised from ethyl acetate to get light brown crystals. Yield 74\% (1.3g). M.p. $=203$ $206^{\circ} \mathrm{C} .{ }^{1} \mathrm{H}-\mathrm{NMR}\left(\mathrm{DMSO}-\mathrm{d}_{6}, 300 \mathrm{MHz}\right) \delta 2.57\left(\mathrm{~s}, 3 \mathrm{H}, \mathrm{CO}_{2} \mathrm{CH}_{3}\right), 3.80\left(\mathrm{~s}, 3 \mathrm{H}, \mathrm{CO}_{2} \mathrm{CH}_{3}\right.$ ), 3.87 (s, $3 \mathrm{H}, \mathrm{CO}_{2} \mathrm{CH}_{3}$ ), 4.49 (s, $2 \mathrm{H}, \mathrm{CH}_{2}$ ), 11.06 (brs, $1 \mathrm{H}, \mathrm{NH}$ ); ${ }^{13} \mathrm{C}-\mathrm{NMR}$ (DMSO-d $\left.d_{6}, 75 \mathrm{MHz}\right) \delta 15.67$ $\left(\mathrm{CH}_{2}\right), 26.83\left(\mathrm{COCH}_{3}\right), 51.71,56.43\left(2 \mathrm{xCO}_{2} \mathrm{CH}_{3}\right), 111.29,114.48,132.58,147.26(4 \mathrm{xC}$, thiophene), $163.84\left(\mathrm{COCH}_{3}\right), 168.04\left(2 \mathrm{xCO}_{2} \mathrm{CH}_{3}\right)$.

5-Acetylamino-3-bromomethylthiophene-2,4-dicarboxylic acid 2-tertbutyl-4-methylester (3c) was prepared by the same procedure as 3c. Yield 67\% (1.32g) of light red crystals M.p. $=$ 92-96 ${ }^{\circ} \mathrm{C} .{ }^{1} \mathrm{H}-\mathrm{NMR}\left(\mathrm{DMSO}-d_{6}, 300 \mathrm{MHz}\right) \delta 1.52\left(\mathrm{~s}, 9 \mathrm{H}, \mathrm{C}\left(\mathrm{CH}_{3}\right)_{3}\right), 2.29\left(\mathrm{~s}, 3 \mathrm{H}, \mathrm{COCH}_{3}\right), 3.02(\mathrm{~s}$, $\left.3 \mathrm{H}, \mathrm{CO}_{2} \mathrm{CH}_{3}\right), 5.18\left(\mathrm{~s}, 2 \mathrm{H}, \mathrm{CH}_{2}\right) ;{ }^{13} \mathrm{C}-\mathrm{NMR}\left(\mathrm{DMSO}-\mathrm{d}_{6}, 75 \mathrm{MHz}\right) \delta 22.9\left(\mathrm{CH}_{2}\right), 27.58\left(\mathrm{C}\left(\mathrm{CH}_{3}\right)_{3}\right)$, $30.1\left(\mathrm{COCH}_{3}\right), 51.91\left(\mathrm{CO}_{2} \mathrm{CH}_{3}\right), 82.23\left(\mathrm{C}\left(\mathrm{CH}_{3}\right)_{3}\right), 128.19,128.92,140.43,150.21(4 \mathrm{xC}$, thiophene), $160.42\left(\mathrm{COCH}_{3}\right), 164.49\left(\mathrm{CO}_{2} \mathrm{CH}_{3}\right), 168.49\left(\mathrm{CO}_{2} \mathrm{C}\left(\mathrm{CH}_{3}\right)_{3}\right)$.

\section{Synthesis of quaternary ammonium salts $4 a-d$. General procedure}

Methyl ester 3a (1g, $3.4 \mathrm{mmol})$ was dissolved in $50 \mathrm{~mL}$ of dry toluene and an equimolar amount of the corresponding tertiary amine $(3.4 \mathrm{mmol})$ was added to a stirred solution. The mixture was left to stir for 24 hours at laboratory temperature. The separated solid was filtrated off and washed with toluene. Gaseous trimethylamine was led directly into the solution of 3a.

1-(5-Acetylamino-2-bromo-4-methoxycarbonylthiophen-3-ylmethyl)-4dimethylaminopyridinium bromide(4a). Yield 91\% (1.52 g), m.p. $=134-135{ }^{\circ} \mathrm{C}{ }^{1} \mathrm{H}-\mathrm{NMR}$ $\left(300 \mathrm{MHz}, \mathrm{CDCl}_{3}\right): \delta: 2.32\left(\mathrm{~s}, 3 \mathrm{H}, \mathrm{COCH}_{3}\right), 3.29\left(\mathrm{~s}, 6 \mathrm{H},\left(\mathrm{CH}_{3}\right)_{2} \mathrm{~N}\right), 4.00\left(\mathrm{~s}, 3 \mathrm{H}, \mathrm{CO}_{2} \mathrm{CH}_{3}\right), 5.66$ (s, $2 \mathrm{H}, \mathrm{CH}_{2}$ ), 7.08 (d, 2H, pyr.), 8.43 (d, 2H, pyr.), 11.25 (s, 1H, NH).

1-[(5-Acetylamino)-2-bromo-4-(methoxycarbonylthiophen-3-yl)methyl]-1,4diaza[2.2.2]bicyclooctyl bromide (4b). Yield $99 \%$ (1.44g), m.p. $=196-198{ }^{\circ} \mathrm{C} .{ }^{1} \mathrm{H}-\mathrm{NMR}$ $\left(300 \mathrm{MHz}, \mathrm{DMSO}-d_{6}\right): \delta: 2.25\left(\mathrm{~s}, 3 \mathrm{H}, \mathrm{COCH}_{3}\right), 3.03\left(\mathrm{~s}, 9 \mathrm{H},\left(\mathrm{CH}_{3}\right)_{3} \mathrm{~N}\right), 4.87\left(\mathrm{~s}, 3 \mathrm{H}, \mathrm{COOCH}_{3}\right)$, $4.66\left(\mathrm{~s}, 2 \mathrm{H}, \mathrm{CH}_{2}\right), 11.00(\mathrm{~s}, 1 \mathrm{H}, \mathrm{NH})$.

1-(5-Acetylamino-2-bromo-4-methoxycarbonyl-3-ylmethyl)-pyridinium bromide (4c). Yield $41 \%$ (0.63 g), m.p. $=201-207{ }^{\circ} \mathrm{C} .{ }^{1} \mathrm{H}$ NMR $\left(300 \mathrm{MHz}, \mathrm{CDCl}_{3}\right): \delta: 2.32\left(\mathrm{~s}, 3 \mathrm{H}, \mathrm{COCH}_{3}\right), 3.93(\mathrm{~s}$, $\left.3 \mathrm{H}, \mathrm{COOCH}_{3}\right), 6.33\left(\mathrm{~s}, 2 \mathrm{H}, \mathrm{CH}_{2}\right), 8.27\left(\mathrm{t}, 2 \mathrm{H}, \mathrm{H}_{3}, \mathrm{H}_{4}\right), 8.68\left(\mathrm{~m}, 1 \mathrm{H}, \mathrm{H}_{5}\right), 9.45\left(\mathrm{~d}, 2 \mathrm{H}, \mathrm{H}_{1}\right), 11.23$ (s, $1 \mathrm{H}, \mathrm{NH})$.

1-(5-Acetylamino-2-bromo-4-methoxycarbonyl-3-ylmethyl)-trimethylamonium bromide (4d). Yield 69 \% (1.13 g), m.p. $=$ subl. $<100{ }^{\circ} \mathrm{C} .{ }^{1} \mathrm{H}-\mathrm{NMR}\left(300 \mathrm{MHz}, \mathrm{DMSO}-d_{6}\right): \delta: 2.34(\mathrm{~s}, 3 \mathrm{H}$, $\left.\mathrm{COCH}_{3}\right), 3.24\left(\mathrm{t}, 6 \mathrm{H},\left(\mathrm{CH}_{2}\right)_{3}\right), 3.72\left(\mathrm{t}, 6 \mathrm{H},\left(\mathrm{CH}_{2}\right)_{3}\right), 3.81\left(\mathrm{~s}, 3 \mathrm{H}, \mathrm{COOCH}_{3}\right), 4.96\left(\mathrm{~s}, 2 \mathrm{H}, \mathrm{CH}_{2}\right)$, $11.24(\mathrm{~s}, 1 \mathrm{H}, \mathrm{NH})$. 
Table 1. UV and IR spectra of quaternary ammonium salts 4a-d

\begin{tabular}{|c|c|c|c|c|c|c|c|}
\hline Compound & $\begin{array}{c}\text { Molecular formula / } \\
\text { Molecular weight }\end{array}$ & $\begin{array}{c}\text { UV (methanol) } \\
\lambda_{\max } / \log \varepsilon\end{array}$ & $\mathrm{COCH}_{3}$ & $\begin{array}{c}\mathrm{IR}, \\
\mathrm{CO}_{2} \mathrm{CH}_{3} \\
\end{array}$ & $\begin{array}{c}(\mathrm{KBr}, v \\
\mathrm{CH}_{2}, \mathrm{CH}_{3}\end{array}$ & $\begin{array}{c}\left., \mathrm{cm}^{-1}\right) \\
\mathrm{C}=\mathrm{C}_{\text {tioph. }}\end{array}$ & $\mathrm{NH}$ \\
\hline $4 a$ & $\begin{array}{c}\mathrm{C}_{16} \mathrm{H}_{19} \mathrm{Br}_{2} \mathrm{~N}_{3} \mathrm{O}_{3} \mathrm{~S} \\
493\end{array}$ & $292.1(5.49), 301.7(4.91)$ & 1576 & 1691 & 2949 & 3150 & 3422 \\
\hline $4 b$ & $\begin{array}{c}\mathrm{C}_{12} \mathrm{H}_{18} \mathrm{Br}_{2} \mathrm{~N}_{2} \mathrm{O}_{3} \mathrm{~S} \\
430\end{array}$ & $226.9(5.18), 301.2(4.87)$ & 1537 & 1700 & 2951 & 3258 & 3473 \\
\hline $4 c$ & $\begin{array}{c}\mathrm{C}_{14} \mathrm{H}_{14} \mathrm{Br}_{2} \mathrm{~N}_{2} \mathrm{O}_{3} \mathrm{~S} \\
450\end{array}$ & $226(5.31), 302.7(4.93)$ & 1539 & 1699 & 2947 & 3271 & 3383 \\
\hline 4d & $\begin{array}{c}\mathrm{C}_{15} \mathrm{H}_{21} \mathrm{Br}_{2} \mathrm{~N}_{3} \mathrm{O}_{3} \mathrm{~S} \\
483\end{array}$ & $226.4(5.22), 300.5(4.92)$ & 1537 & 1696 & 2949 & 3267 & 3379 \\
\hline
\end{tabular}

Synthesis of thiouronium salts 5a-c. General procedure

Bromomethyl derivates $(5 \mathrm{mmol})$ and thiourea $(0.84 \mathrm{~g}, 11 \mathrm{mmol})$ in $20 \mathrm{~mL}$ dry acetone were refluxed under inert atmosphere of argon for 5-10 hrs. The reaction mixture was cooled down do room temperature and left to stay for all night. Precipitated solid was then filtered off, filtrate was concentrated under vacuum, the residue was combined with precipitated and crystallised from methanol to yield thiouronium salts 5a-c.

1-(5-Acetylamino-2-bromo-4-methoxycarbonyl-3-ylmethyl)-thiouronium bromide (5a). Yield $95 \%(2 \mathrm{~g})$ of white powdered product. M.p. $=194-198^{\circ} \mathrm{C} .{ }^{1} \mathrm{H}-\mathrm{NMR}\left(\mathrm{DMSO}-d_{6}, 300 \mathrm{MHz}\right) \delta$ 3.30 (s, $3 \mathrm{H}, \mathrm{COCH}_{3}$ ), 3.88 (s, $3 \mathrm{H}, \mathrm{CO}_{2} \mathrm{CH}_{3}$ ), 4.57 (s, $2 \mathrm{H}, \mathrm{CH}_{2}$ ), 9.19 (br s, $\left.4 \mathrm{H}, 2 \mathrm{xNH}_{2}\right), 10.96$ (s, $1 \mathrm{H}, \mathrm{NH}) ;{ }^{13} \mathrm{C}-\mathrm{NMR}\left(\mathrm{DMSO}_{6}, 75 \mathrm{MHz}\right) \delta 22.69\left(\mathrm{CH}_{2}\right), 27.6\left(\mathrm{C}\left(\mathrm{CH}_{3}\right)_{3}\right), 30.2\left(\mathrm{COCH}_{3}\right), 52.14$ $\left(\mathrm{CO}_{2} \mathrm{CH}_{3}\right), 109.96,109.97,129.48,148.16\left(4 \mathrm{xC}\right.$, thiophene), $162.71\left(\mathrm{COCH}_{3}\right), 168.61$ $\left(\mathrm{CO}_{2} \mathrm{CH}_{3}\right)$.

1-(5-Acetylamino-2,4-dimethoxycarbonyl-3-ylmethyl)thiouronium bromide (5b). Yield 91\% $(1.91 \mathrm{~g})$ of white powdered product. M.p. $=168-171^{\circ} \mathrm{C} .{ }^{1} \mathrm{H}-\mathrm{NMR}\left(\mathrm{DMSO}-\mathrm{d}_{6}, 300 \mathrm{MHz}\right) \delta 2.03(\mathrm{~s}$, $\left.3 \mathrm{H}, \mathrm{COCH}_{3}\right), 3.78\left(\mathrm{~s}, 3 \mathrm{H}, \mathrm{CO}_{2} \mathrm{CH}_{3}\right), 3.84\left(\mathrm{~s}, 3 \mathrm{H}, \mathrm{CO}_{2} \mathrm{CH}_{3}\right), 4.88\left(\mathrm{~s}, 2 \mathrm{H}, \mathrm{CH}_{2}\right), 9.03$ (brs, $4 \mathrm{H}$, $\left.2 \mathrm{xNH}_{2}\right), 11.02$ (brs, $\left.1 \mathrm{H}, \mathrm{NH}\right) ;{ }^{13} \mathrm{C}-\mathrm{NMR}$ (DMSO-d $\left.6,75 \mathrm{MHz}\right) \delta 22.69\left(\mathrm{CH}_{2}\right), 29.41\left(\mathrm{COCH}_{3}\right)$, $51.15\left(2 \mathrm{xCO}_{2} \mathrm{CH}_{3}\right), 109.96,109.97,129.48,148.16\left(4 \mathrm{xC}\right.$, thiophene), $162.71\left(\mathrm{COCH}_{3}\right), 168.61$, $169.31\left(2 \mathrm{xCO}_{2} \mathrm{CH}_{3}\right)$.

1-(5-Acetylamino-2-tertbutoxycarbonyl-4-methoxycarbonyl-3-ylmethyl) thiouronium bromide (5c). Yield $94 \%(2.14 \mathrm{~g})$ of white powdered product. M.p. $=48-51{ }^{\circ} \mathrm{C} .{ }^{1} \mathrm{H}-$ NMR(DMSO- $\left.d_{6}, 300 \mathrm{MHz}\right) \delta 1.54\left(\mathrm{~s}, 9 \mathrm{H}, \mathrm{C}\left(\mathrm{CH}_{3}\right)_{3}\right), 2.30\left(\mathrm{~s}, 3 \mathrm{H}, \mathrm{CH}_{3}\right), 3.89\left(\mathrm{~s}, 3 \mathrm{H}, \mathrm{CO}_{2} \mathrm{CH}_{3}\right)$, 4.93 (s, $2 \mathrm{H}, \mathrm{CH}_{2}$ ), 9.07 (brs, $2 \mathrm{H}, 2 \mathrm{xNH}_{2}$ ), 11.02 (brs, $1 \mathrm{H}, \mathrm{NH}$ ) $;{ }^{13} \mathrm{C}$ NMR (DMSO-d 6 , $75 \mathrm{MHz}$ ) $\delta$ $22.92\left(\mathrm{CH}_{2}\right), 30.10\left(\mathrm{COCH}_{3}\right), 52.61\left(\mathrm{CO}_{2} \mathrm{CH}_{3}\right), 81.36\left(\mathrm{C}_{\left.\left(\mathrm{CH}_{3}\right)_{3}\right), 106.07,109.98,129.48,148.16}\right.$ (4xC, thiophene), $162.72\left(\mathrm{COCH}_{3}\right), 168.61\left(\mathrm{CO}_{2} \mathrm{CH}_{3}\right), 169.32\left(\mathrm{CO}_{2} \mathrm{C}\left(\mathrm{CH}_{3}\right)_{3}\right)$.

Hydrolysis of the thiouronium salt $5 \mathrm{a}$ - synthesis of the 2-acetylamino-5-bromo-4tiomethylthiophene-3-carboxylic acid (6a)

Thiouronium salt $5 a$ a $(0.5 \mathrm{~g}, 1.1 \mathrm{mmol})$ and methanolic $\mathrm{KOH}(0.49 \mathrm{~g} \mathrm{KOH}$ dissolved in $4.4 \mathrm{ml}$ of 1:1 aqueous methanol) was refluxed for $1 \mathrm{~h}$, methanol was distilled off and the flask cooled to 0 - 
$5{ }^{\circ} \mathrm{C}$, acidified by $1 \mathrm{M} \mathrm{HCl}$ and extracted with ether 3 times. Ether extracts were combined, dried over with sodium sulphate and evaporated to dryness. Yield $61 \%(0.21 \mathrm{~g})$ of grey powdered product was obtained, For $\mathrm{C}_{9} \mathrm{H}_{10} \mathrm{BrNO}_{3} \mathrm{~S}_{2}$

(324) m.p. $=163-167{ }^{\circ} \mathrm{C} .{ }^{1} \mathrm{H}-\mathrm{NMR}\left(\mathrm{CDCl}_{3}, 300 \mathrm{MHz}\right) \delta 2.08(\mathrm{t}, 1 \mathrm{H}, \mathrm{SH}), 2.16\left(\mathrm{~s}, 3 \mathrm{H}, \mathrm{COCH}_{3}\right)$, $4.11\left(\mathrm{~d}, 2 \mathrm{H}, \mathrm{CH}_{2}\right), 9.50(\mathrm{~s}, 1 \mathrm{H}, \mathrm{COOH}), 11.27$ (s, 1H, NH).

Attempt at cyclization of thiouronium salts - synthesis of $\mathrm{N}$-(3-bromo-6-oxo-4H,6Hthieno[3,4-c]thiophene-1-yl)acetamide (7a)

Thiouronium salt (5a) (1g, $2.2 \mathrm{mmol})$ was hydrolysed by heating $3 \mathrm{hrs}$. at boiling water bath by aqueous $\mathrm{NaHCO}_{3}\left(0.19 \mathrm{~g} \mathrm{NaHCO}_{3}\right.$ in $75 \mathrm{~mL}$ of distilled water $)$. After cooling the separated light yellow solid was filtered off to give $51 \%$ (0.4g approx.) a mixture of three compounds $\mathbf{6 a}, 7 \mathbf{a}$ and 8a in ratio 22\% : 19\% : 10\% separated by flash column liquid chromatography (eluent 20\% AcOEt in $i$-hexane).

2-Acetylamino-5-bromo-4sulfanylmethylthiophene-3-carboxylic acid (6a). Yield $22 \%$ (0.22 g), m.p. $=163-167{ }^{\circ} \mathrm{C}$. For $\mathrm{C}_{8} \mathrm{H}_{8} \mathrm{BrNO}_{3} \mathrm{~S}_{2}\left(\mathrm{M}_{\mathrm{r}}=310.18\right) .{ }^{1} \mathrm{H}-\mathrm{NMR}\left(\mathrm{CDCl}_{3}, 300 \mathrm{MHz},\right) \delta 2.08(\mathrm{t}$, $1 \mathrm{H}, \mathrm{SH}), 2.16\left(\mathrm{~s}, 3 \mathrm{H}, \mathrm{COCH}_{3}\right), 4.11\left(\mathrm{~d}, 2 \mathrm{H}, \mathrm{CH}_{2}\right), 9.50\left(\mathrm{~s}, 1 \mathrm{H}, \mathrm{CO}_{2} \mathrm{H}\right), 11.27(\mathrm{~s}, 1 \mathrm{H}, \mathrm{NH})$.

$\boldsymbol{N}$-(3-Bromo-6-oxo-4H,6H-thieno[3,4-c]thiophene-1-yl)acetamide (7a). Yield $19 \%$ (0.095 g), m.p. $=187-190{ }^{\circ} \mathrm{C}$. For $\mathrm{C}_{8} \mathrm{H}_{6} \mathrm{BrNO}_{2} \mathrm{~S}_{2}\left(\mathrm{M}_{\mathrm{r}}=292.16\right) .{ }^{1} \mathrm{H}-\mathrm{NMR}\left(300 \mathrm{MHz}, \mathrm{CDCl}_{3}\right)$ : $\delta: 2.16(\mathrm{~s}$, $\left.3 \mathrm{H}, \mathrm{COCH}_{3}\right), 4.26\left(\mathrm{~d}, 2 \mathrm{H}, \mathrm{CH}_{2}\right), 11.27(\mathrm{~s}, 1 \mathrm{H}, \mathrm{NH})$.

2- $N$-acetylamino-5-bromo-4-mercaptomethyl-3-carbometoxythiophene (6). Yield $9.8 \%$ (0.05g), m.p. $=117-119{ }^{\circ} \mathrm{C}$. For $\mathrm{C}_{9} \mathrm{H}_{10} \mathrm{BrNO}_{3} \mathrm{~S}_{2}\left(\mathrm{M}_{\mathrm{r}}=324\right) .{ }^{1} \mathrm{H}-\mathrm{NMR}\left(\mathrm{CDCl}_{3}, 300 \mathrm{MHz}\right): \delta: 2.01$ (d, 1H, SH), $2.28\left(\mathrm{~s}, 3 \mathrm{H}, \mathrm{COCH}_{3}\right), 3.89$ (s, 3H, $\left.\mathrm{CO}_{2} \mathrm{CH}_{3}\right), 4.09\left(\mathrm{~s}, 2 \mathrm{H}, \mathrm{CH}_{2}\right), 10.11(\mathrm{~s}, 1 \mathrm{H}, \mathrm{NH})$.

Cyclization of thiouronium salts - synthesis of thieno[3,4-c]thiolactones 7a-c, general procedure

Thiouronium salt (6a-c) $(2.1 \mathrm{mmol})$ was treated with $50 \mathrm{~mL}$ methanolic solution of $\mathrm{NaHCO}_{3}(2.1 \mathrm{~g}$ of $\mathrm{NaHCO}_{3}$ in $50 \%$ aqueous methanol). The reaction mixture was stirred at $70-80^{\circ} \mathrm{C}$ under inert argon atmosphere for $5 \mathrm{hrs}$ until $\mathrm{CO}_{2}$ completely removed from the mixture. After cooling down to laboratory temperature the solid was filtered off, washed with diluted $\mathrm{HCl}$, dried up to yield appropriate thieno[3,4-c] thiolactone derivate.

$\boldsymbol{N}$-(3-Bromo-6-oxo-4H,6H-thieno[3,4-c]thiophene-1-yl)acetamide (7a). Yield $46 \%(0.28 \mathrm{~g})$ of light grey solid product. M.p. $=187-190^{\circ} \mathrm{C} .{ }^{1} \mathrm{H}-\mathrm{NMR}\left(\mathrm{DMSO}-\mathrm{d}_{6}, 300 \mathrm{MHz}\right) \delta 3.24(\mathrm{~s}, 3 \mathrm{H}$, $\left.\mathrm{COCH}_{3}\right), 4.2\left(\mathrm{~s}, 2 \mathrm{H}, \mathrm{CH}_{2}\right), 11.05$ (brs, $\left.1 \mathrm{H}, \mathrm{NH}\right) ;{ }^{13} \mathrm{C}-\mathrm{NMR}\left(\mathrm{DMSO}-d_{6}, 75 \mathrm{MHz}\right) \delta 22.1\left(\mathrm{CH}_{2}\right)$, $29.23\left(\mathrm{COCH}_{3}\right), 95.15,122.21,122.24,140.92(4 \mathrm{xC}$, thiophene $), 169.13\left(\mathrm{COCH}_{3}\right), 187.04(\mathrm{C}=\mathrm{O}$ thiolactone).

3-Acetylamino-4-oxo-4H,6H-thieno[3,4-c]thiopehene-1-carboxylic acid methyl ester (7b). Yield $48 \%(0.27 \mathrm{~g})$ light yellow product. M.p. $=187-190^{\circ} \mathrm{C} .{ }^{1} \mathrm{H}-\mathrm{NMR}\left(\mathrm{DMSO}-d_{6}, 300 \mathrm{MHz}\right) \delta$ 2.03 (s, 3H, $\left.\mathrm{COCH}_{3}\right), 3.71$ (s, 3H, $\left.\mathrm{CO}_{2} \mathrm{CH}_{3}\right), 4.45$ (s, $\left.2 \mathrm{H}, \mathrm{CH}_{2}\right), 8.30$ (brs, $\left.1 \mathrm{H}, \mathrm{NH}\right) ;{ }^{13} \mathrm{C} \mathrm{NMR}$ $\left(\mathrm{DMSO}_{6}, 75 \mathrm{MHz}\right) \delta 22.02\left(\mathrm{CH}_{2}\right), 34.61\left(\mathrm{COCH}_{3}\right), 51.16\left(\mathrm{CO}_{2} \mathrm{CH}_{3}\right), 102.23,116.47,129.4$, 141.5 (4xC, thiophene), $160.87\left(\mathrm{COCH}_{3}\right), 163.81\left(\mathrm{CO}_{2} \mathrm{CH}_{3}\right), 186.46(\mathrm{C}=\mathrm{O}$ thiolactone $)$. 
3-Acetylamino-4-oxo-4H,6H-thieno[3,4-c]thiopehene-1-carboxylic acid tert-butyl ester (7c). Yield 51\% (0.33g) light grey solid product. M.p. $=187-190{ }^{\circ} \mathrm{C} .{ }^{1} \mathrm{H}$ NMR $\left(\mathrm{DMSO}-d_{6}, 300 \mathrm{MHz}\right) \delta$ $1.48\left(\mathrm{~s}, 9 \mathrm{H}, \mathrm{C}\left(\mathrm{CH}_{3}\right)_{3}\right), 4.35\left(\mathrm{~s}, 2 \mathrm{H}, \mathrm{CH}_{2}\right), 8.03$ (brs, $\left.1 \mathrm{H}, \mathrm{NH}\right) ;{ }^{13} \mathrm{C}$ NMR (DMSO-d 6 , 75MHz) $\delta$

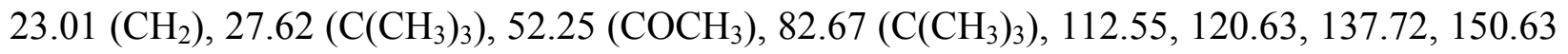
(4xC, thiophene), $169.70\left(\mathrm{CO}_{2} \mathrm{C}\left(\mathrm{CH}_{3}\right)_{3}\right), 183.79(\mathrm{C}=\mathrm{O}$ thiolactone $)$.

Table 2: Molecular formula, molecular weight and IR spectra of compounds 3a-c, 5a-7c

\begin{tabular}{|c|c|c|c|c|c|c|c|c|c|}
\hline Comp. & $\begin{array}{c}\text { Molecular } \\
\text { formula / } \\
\text { Molecular weight }\end{array}$ & $\mathrm{CH}_{2}-\mathrm{X}$ & $\begin{array}{l}\mathrm{C}=\mathrm{C} \\
\text { tioph. }\end{array}$ & $\begin{array}{c}\mathrm{IR}, \\
\mathrm{COCH}_{3}\end{array}$ & $\begin{array}{l}(\mathrm{KBr}, \quad v \\
\mathrm{CO}_{2} \mathrm{CH}_{3}\end{array}$ & $\begin{array}{c}\left.\mathrm{cm}^{-1}\right) \\
\mathrm{CO}_{2} t \mathrm{Bu}\end{array}$ & $\mathrm{NH}$ & $\mathrm{NH}_{2}$ & $\begin{array}{l}\mathrm{C}=\mathrm{O} \\
\text { tiolact. }\end{array}$ \\
\hline 3a & $\begin{array}{c}\mathrm{C}_{9} \mathrm{H}_{9} \mathrm{Br}_{2} \mathrm{NO}_{3} \mathrm{~S} \\
371\end{array}$ & $\begin{array}{c}569 \\
\mathrm{X}=\mathrm{Br}\end{array}$ & $\begin{array}{l}1439 \\
1502 \\
1542\end{array}$ & 1675 & 1695 & - & 3246 & - & - \\
\hline $3 \mathbf{b}$ & $\begin{array}{c}\mathrm{C}_{11} \mathrm{H}_{12} \mathrm{BrNO}_{3} \mathrm{~S} \\
350\end{array}$ & $\begin{array}{c}579 \\
\mathrm{X}=\mathrm{Br}\end{array}$ & $\begin{array}{l}1434 \\
1501 \\
1544\end{array}$ & 1676 & $\begin{array}{l}1694 \\
1707\end{array}$ & - & 3247 & - & - \\
\hline $3 c$ & $\begin{array}{c}\mathrm{C}_{14} \mathrm{H}_{18} \mathrm{BrNO}_{3} \mathrm{~S} \\
392\end{array}$ & $\begin{array}{c}572 \\
\mathrm{X}=\mathrm{Br}\end{array}$ & $\begin{array}{l}1441 \\
1505 \\
1542\end{array}$ & 1682 & 1692 & 1701 & 3253 & - & - \\
\hline $5 a$ & $\begin{array}{c}\mathrm{C}_{9} \mathrm{H}_{13} \mathrm{Br}_{2} \mathrm{~N}_{3} \mathrm{O}_{3} \mathrm{~S}_{2} \\
435\end{array}$ & $\begin{array}{l}1410 \\
X=S\end{array}$ & $\begin{array}{l}1432 \\
1449 \\
1539\end{array}$ & 1673 & 1690 & - & 3250 & $\begin{array}{l}3169 \\
3370\end{array}$ & - \\
\hline $5 b$ & $\begin{array}{c}\mathrm{C}_{11} \mathrm{H}_{16} \mathrm{BrN}_{3} \mathrm{O}_{3} \\
414\end{array}$ & $\begin{array}{l}1413 \\
X=S\end{array}$ & $\begin{array}{l}1436 \\
1502 \\
1549\end{array}$ & 1675 & 1695 & - & 3277 & $\begin{array}{l}3176 \\
3373\end{array}$ & - \\
\hline $5 c$ & $\begin{array}{c}\mathrm{C}_{14} \mathrm{H}_{22} \mathrm{BrN}_{3} \mathrm{O}_{3} \mathrm{~S}_{2} \\
456\end{array}$ & $\begin{array}{l}1414 \\
X=S\end{array}$ & $\begin{array}{l}1442 \\
1502 \\
1544\end{array}$ & 1649 & 1676 & 1612 & 3275 & $\begin{array}{l}3175 \\
3382\end{array}$ & - \\
\hline $7 a$ & $\begin{array}{c}\mathrm{C}_{8} \mathrm{H}_{6} \mathrm{BrNO}_{2} \mathrm{~S}_{2} \\
292\end{array}$ & $\begin{array}{l}1405 \\
X=S\end{array}$ & $\begin{array}{l}1436 \\
1501 \\
1539\end{array}$ & 1671 & - & - & 3278 & - & 1620 \\
\hline $7 b$ & $\begin{array}{c}\mathrm{C}_{10} \mathrm{H}_{9} \mathrm{NO}_{4} \mathrm{~S}_{2} \\
271\end{array}$ & $\begin{array}{l}1407 \\
X=S\end{array}$ & $\begin{array}{l}1431 \\
1477 \\
1528\end{array}$ & 1637 & 1695 & - & 3249 & - & 1670 \\
\hline $7 c$ & $\begin{array}{c}\mathrm{C}_{13} \mathrm{H}_{15} \mathrm{NO}_{4} \mathrm{~S}_{2} \\
313\end{array}$ & $\begin{array}{l}1408 \\
X=S\end{array}$ & $\begin{array}{l}1460 \\
1510 \\
1545 \\
\end{array}$ & 1644 & - & 1682 & 3274 & - & 1682 \\
\hline
\end{tabular}

\section{Acknowledgements}


We thank to the Slovak Grant Agency, Slovak republic for financial support of this work (Grant $\mathrm{N}^{\mathrm{o}}$ : 1/1379/04) and the Science and Technology Assistance Agency (contract No. APVT-20007304). The authors are grateful to Mrs. Silvia Markusová for IR spectra measurements and to Dr. Nad'a Prónayová for NMR spectra measurements. ZP appreciates to Prof. Dr. Alžbeta Krutošíková and Ing. Peter Zálupský PhD. for correcting the manuscript.

\section{References and Footnotes}

1. Lecrec, M.; Ho, H., A. Synlett 2004, 2, 380.

2. Bednarz, M.; Reineker, P.; E., Mena-Osteriz; Bäuerle, P. J. of Luminiscence 2004, 110, 225.

3. Pisignano, D.; Della Sala, F.; Persano, L.; Gigli, G.; Cingolani, R.; Barbarella, G.; Favretto, L. Physica A 2004, 339, 106.

4. Lukeš, V.; Breza, M.; Végh, D.; Hrdlovič, P; Laurinc, V. Synth. Metals 2003, 138, 399.

5. Amaresh, R., R.; Lakshmikantham, M., V.; Baldwin J., W.; Cava, M., P.; Metzger, R., M.; Rogers, R., D. J. Org. Chem. 2002, 67, 2453.

6. Ishii, A.; Ida, Y.; Nakayama, J.; Hoshino, M. Bull. Chem. Soc. Jpn. 1992, 65, 2821.

7. Miller, K., J.; Moschner, K., F.; Potts, K., T. J. Am. Chem. Soc. 1983, 105, 785.

8. Gewald K. Angew. Chem. 1961, 73, 114.

9. Pavlovičová, R.; Mináriková, J.; Hudecová, D.; Végh, D. Chem. Papers 1997, 51, 437.

10. Gudriniece, E.; Pālītis, Ē; Barkāne, V. Latvijas AV. Izv. AN Lat. SSR 1983, 5, 614.

11. Végh, D.; Morel, J.; Decroix, B.; Zálupský, P. Synth. Commun. 1992, 22, 2057.

12. Pal, R.; Murty, K., V. S., N., Mal, D. Synth. Commun. 1993, 23, 1555.

13. All new compounds have correct $\mathrm{C}, \mathrm{H}, \mathrm{N}$ elemental analyses. 